\title{
Avaliação do decaimento de amplitude de ondas sísmicas em meios compostos de camadas plano estratificadas.
}

Jozinei Ferreira Lopes, Universidade Federal do Pará - UFPA

Raimundo Nonato Colares Carneiro, Universidade Federal do Oeste do Pará - UFOPA

Jorge Kysnney Santos Kamassury, Universidade Federal de Santa Catarina - UFSC

Marlon de Sousa Mota, Universidade Federal do Pará - UFPA

Copyright 2018, SBGf - Sociedade Brasileira de Geofísica

Este texto foi preparado para a apresentação no VIII Simpósio Brasileiro de Geofísica, Salinópolis, 18 a 20 de setembro de 2018. Seu conteúdo foi revisado pelo Comitê Técnico do VIII SimBGf, mas não necessariamente representa a opinião da SBGf ou de seus associados. É proibida a reprodução total ou parcial deste material para propósitos comerciais sem prévia autorização da SBGf.

\begin{abstract}
The present work had the objective of analyzing the decay of seismic wave amplitude in media composed of homogeneous layers and flat-stratified analyzing the cases of reflection and transmission under conditions of normal and oblique incidence. For this, a geological model composed of twelve layers composed by a realistic speed distribution was considered, aiming to obtain values of transit time of the seismic wave; transmission and reflection coefficients in normal and oblique incidence, spherical divergence, cumulative amplitude deviation of descending and ascending waves, and finally the construction of the seismogram resulting from the interaction of the wave with the means to observe in what way the amplitude of the signal attenuates as the wave strikes the deepest interfaces.
\end{abstract}

\section{Introdução}

O método sísmico de reflexão estuda as propriedades físicas das rochas em subsuperfície através da propagação de ondas sísmicas no interior destas, sofrendo eventos de reflexão e transmissão à medida que incidem nas interfaces entre as diferentes camadas. Ao refletirem, as ondas retornam à superfície e são captadas por um conjunto de sensores e os tempos de reflexão são gravados na forma de sismogramas. Ao transmitirem, por sua vez, estas ondas se propagam em camadas mais profundas, sofrendo novas reflexões e transmissões enquanto tiverem energia suficiente, haja vista que perdem energia devido à divergência esférica. $O$ objetivo central deste trabalho consiste em relacionar essa perda de energia da onda sísmica com a variação dos valores de divergência esférica entre as camadas, descrevendo a propagação de ondas sísmicas em um modelo de camadas plano-estratificados e calculando, para os casos de incidência normal e oblíqua, os coeficientes de (i) desvio de amplitude acumulada para a onda descendente da primeira para a i-ésima camada, no caso de incidência normal e oblíqua, (ii) coeficiente de desvio de amplitude acumulada para a onda ascendente da i-ésima para a primeira camada, no caso de incidência normal e oblíqua e (iii) matriz refletividade generalizada, que fornece a disposição espacial dos pulsos sísmicos, viabilizando a geração do sismograma resultante das interações da onda com as diferentes interfaces.

\section{Metodologia}

Inicialmente, consideremos um modelo com $n$ camadas planas e horizontais, com velocidades e densidades dadas por $v_{i}$ e $\rho_{i}(i=1, \ldots, 12)$.

O cálculo do afastamento fonte-sensor $x(p)$ e do tempoduplo $t(x)$ são propriamente expressos em termos do parâmetro horizontal do raio $(p)$ nas formas:

$x(p)=2 p \sum_{i=1}^{n} \frac{v_{i}^{2} \Delta t_{i}}{\sqrt{1-p^{2} v_{i}^{2}}}, \quad t(p)=2 \sum_{i=1}^{n} \frac{\Delta t_{i}}{\sqrt{1-p^{2} v_{i}^{2}}}$

$\mathrm{Na}$ combinação $x(p)$ e $\mathrm{t}(p)$, o fator de divergência esférica, mostrado na Figura 1, é dado por (NEWMAN, 1973):

$R_{0}(x)=\frac{\sqrt{1-p^{2} v_{0}^{2}}}{p v_{0}}\left[x^{2}+2 x \sum_{i=1}^{n} e_{i}\left(\frac{p v_{i}}{\sqrt{1-p^{2} v_{i}^{2}}}\right)^{3}\right]^{1 / 2}$

O espalhamento por transmissão-reflexão num meio plano-estratificado é outro conceito importante deste modelo para interpretação de dados observados, além da parte cinemática pura, e no cálculo de amplitudes como apresentado por (AKI; RICHARDS, 1980-2002) e (SANDMEIER, 1990) com o método da refletividade; e além disso, pode-se citar (ROBINSON; TREITEL, 2000) voltado à propagação em camadas finas. Isto quer dizer que a interpretação de seções sísmicas necessita de justificativas baseadas em modelos sintéticos. Com esta finalidade, considerando a propagação de ondas planas ao longo do raio traçado na Figura 1, o cálculo dos coeficientes de reflexão $\left(R_{n}\right)$ e transmissão $\left(T_{n}\right)$ no caso de normal incidência são dados por (BARTOLO L.; DORS, 2011):

$R_{n}=\frac{1-\alpha_{n} \beta_{n}}{1+\alpha_{n} \beta_{n}}$ e $T_{n}=\frac{2}{1+\alpha_{n} \beta_{n}}$

onde $\alpha_{n}=\rho_{n} / \rho_{n+1}$ e $\beta_{n}=v_{n} / V_{n+1}$.

O coeficiente de transmissão acumulado da onda descendente correspondente à razão entre a amplitude da onda normal gerada na primeira camada e a amplitude da onda normal que chega na i-ésima camada é dado por $A_{i}$; enquanto, o coeficiente de transmissão acumulado da onda ascendente dado por $B_{i}$, por sua vez, corresponde à razão entre a amplitude da onda refletida na i-ésima camada e a amplitude da onda normal que retorna para a primeira camada.

$$
A_{i}=2 \prod_{j=1}^{i-1} \frac{1}{1+\alpha_{j} \beta_{j}}, B_{i}=4\left(\frac{1-\alpha_{i} \beta_{i}}{1+\alpha_{i} \beta_{i}}\right) \prod_{j=1}^{i-1} \frac{1}{\left(1+\alpha_{j} \beta_{j}\right)^{2}}
$$


Os coeficientes $A_{i}$ e $B_{i}$ são desenhados na Figura 2.
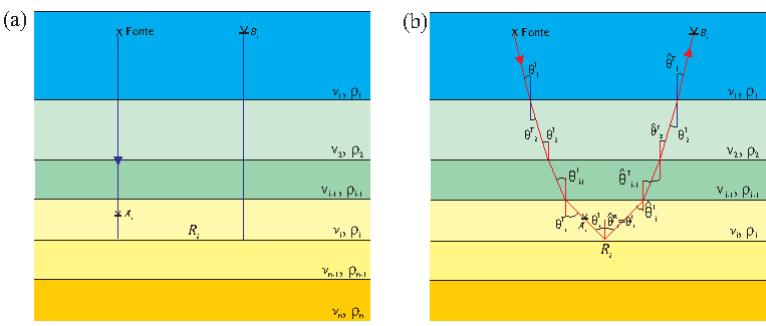

Figura 1 - Em (a) o raio de onda incide perpendicularmente na interface. Em (b) o raio incide obliquamente nas interfaces. São mostrados os caminhos dos raios descendentes e ascendentes. Em (b) são mostrados os ângulos de incidência $\theta^{i} e$ transmissão $\theta^{T}$ para os raios descendentes $\left(\theta_{i}\right)$ e $\left(\hat{\theta}_{i}\right)$.

Além disso, o desvio de amplitude acumulada pela onda normal descendente na i-ésima camada é denotado por $r_{i}^{A}$ e o desvio de amplitude acumulada pela onda normal ascendente da i-ésima até a superfície é dado por $r_{i}^{B}$.

$r_{i}^{A}=\prod_{j=1}^{i-1} \frac{1+\alpha_{j} \beta_{j}}{1+\beta_{j}}, r_{i}^{B}=\left(\frac{1+\alpha_{i} \beta_{i}}{1+\beta_{i}}\right)\left(\frac{1-\beta_{i}}{1-\alpha_{i} \beta_{i}}\right) \prod_{j=1}^{i-1} \frac{\left(1+\alpha_{j} \beta_{j}\right)^{2}}{\alpha_{j}\left(1+\beta_{j}\right)^{2}}$

No caso de incidência oblíqua (Figura 1), os coeficientes de reflexão e transmissão são dados por $\bar{R}_{i}$ e $\bar{T}_{i}$, respectivamente.

$\bar{R}_{i}=\frac{1-\alpha_{i} \beta_{i} \gamma_{i}}{1+\alpha_{i} \beta_{i} \gamma_{i}}, \bar{T}_{i}=\frac{2}{1+\alpha_{i} \beta_{i} \gamma_{i}}, \theta_{i+1}^{T}=\operatorname{arcsen}\left(\frac{\operatorname{sen} \theta_{i}^{T}}{\beta_{i}}\right)$

Acentua-se que os ângulos de transmissão e incidência se relacionam através de $\theta_{i+1}^{T}$.

Os desvios de amplitude acumulada nos casos de incidências oblíquas, devido a onda descendente na iésima camada e devido a onda ascendente até a superfície são, nessa ordem, dados pelos coeficientes das pelas Eqs. (8) e (9):

$\bar{r}_{i}^{A}=\prod_{j=1}^{i-1} \frac{1+\alpha_{j} \beta_{j} \gamma_{j}}{1+\beta_{j} \gamma_{j}}$

$\bar{r}_{i}^{B}=\left(\frac{1+\alpha_{i} \beta_{i} \gamma_{i}}{1+\beta_{i} \gamma_{i}}\right)\left(\frac{1-\beta_{i} \gamma_{i}}{1-\alpha_{i} \beta_{i} \gamma_{i}}\right) \prod_{j=1}^{i-1} \frac{\left(1+\alpha_{j} \beta_{j} \gamma_{j}\right)^{2}}{\alpha_{j}\left(1+\beta_{j} \gamma_{j}\right)^{2}}$

\section{Resultados}

Nesta seção será feita a análise dos coeficientes $\bar{r}_{i}^{A}$ (em profundidade) e $\bar{r}_{i}^{B}$ (na superfície) para um modelo de doze camadas, conforme apresentado na Tabela (1).

Os parâmetros da aquisição de nosso modelo são descritos na Tabela 2.

Um dos conceitos básicos da propagação de ondas é a divergência esférica responsável pelo decaimento do campo e, consequentemente, a dificuldade de registro. Sendo assim, nesta seção apresentamos detalhes das etapas da modelagem sísmica ilustradas no
Fluxograma 1, além da descrição do modelo proposto para realização das referidas etapas, com o objetivo de mostrar formas de divergência esférica de ondas primárias a partir de fontes pontuais em modelos planohorizontais, com distribuição variável de velocidade, simulando o modelo oceânico.

Tabela 1: Modelo de doze camadas, com diferentes densidades $\left(\mathrm{em} \mathrm{kg} / \mathrm{m}^{3}\right)$ e modelos de velocidades (em $\mathrm{m} / \mathrm{s}$ ). Os perfis de densidade e de velocidade são valores típicos de modelos reais marinhos.

\begin{tabular}{|l|c|c|}
\hline \multicolumn{3}{|c|}{ Modelo } \\
\hline camada & densidade & velocidade \\
\hline 1 & 1000 & 1500 \\
\hline 2 & 1150 & 1762 \\
\hline 3 & 1250 & 2002 \\
\hline 4 & 1350 & 2221 \\
\hline 5 & 1450 & 2420 \\
\hline 6 & 1550 & 2602 \\
\hline 7 & 1650 & 2769 \\
\hline 8 & 1750 & 2920 \\
\hline 9 & 1850 & 3059 \\
\hline 10 & 1950 & 3185 \\
\hline 11 & 2050 & 3300 \\
\hline 12 & 2150 & 3405 \\
\hline
\end{tabular}

Tabela 2: Parâmetros da aquisição do dado sintético.

\begin{tabular}{|c|c|}
\hline \multicolumn{2}{|c|}{ Geometria de Fontes e Receptores } \\
\hline Número de Fontes & 1 \\
\hline $\begin{array}{c}\text { Número de } \\
\text { Receptores }\end{array}$ & 12 \\
\hline $\begin{array}{c}\text { Intervalo entre } \\
\text { Receptores }\end{array}$ & $100 \mathrm{~m}$ \\
\hline Offset mínimo & $300 \mathrm{~m}$ \\
\hline Offset máximo & $1400 \mathrm{~m}$ \\
\hline Lanço de geofones & $300 \mathrm{a} 1400 \mathrm{~m}$ \\
\hline
\end{tabular}

\begin{tabular}{|c|c|}
\hline \multicolumn{2}{|c|}{ Geometria do Modelo } \\
\hline Número de Camadas & 12 \\
\hline $\begin{array}{c}\text { Profundidade da primeira } \\
\text { camada }\end{array}$ & $200 \mathrm{~m}$ \\
\hline $\begin{array}{c}\text { Profundidade da segunda } \\
\text { camada } \\
\end{array}$ & $602 \mathrm{~m}$ \\
\hline $\begin{array}{c}\text { Profundidade da terceira } \\
\text { camada }\end{array}$ & $1008 \mathrm{~m}$ \\
\hline $\begin{array}{l}\text { Profundidade da quarta } \\
\text { camada }\end{array}$ & $1430 \mathrm{~m}$ \\
\hline $\begin{array}{c}\text { Profundidade da quinta } \\
\text { camada }\end{array}$ & $1848 \mathrm{~m}$ \\
\hline $\begin{array}{l}\text { Profundidade da sexta } \\
\text { camada }\end{array}$ & $2270 \mathrm{~m}$ \\
\hline $\begin{array}{c}\text { Profundidade da sétima } \\
\text { camada }\end{array}$ & $1696 \mathrm{~m}$ \\
\hline $\begin{array}{c}\text { Profundidade da oitava } \\
\text { camada }\end{array}$ & $3126 \mathrm{~m}$ \\
\hline $\begin{array}{l}\text { Profundidade da nona } \\
\text { camada }\end{array}$ & $3650 \mathrm{~m}$ \\
\hline $\begin{array}{c}\text { Profundidade da décima } \\
\text { camada }\end{array}$ & $3998 \mathrm{~m}$ \\
\hline $\begin{array}{c}\text { Profundidade da décima } \\
\text { primeira camada }\end{array}$ & $4440 \mathrm{~m}$ \\
\hline $\begin{array}{l}\text { Profundidade da décima } \\
\text { segunda camada }\end{array}$ & $4886 \mathrm{~m}$ \\
\hline
\end{tabular}

\begin{tabular}{|c|c|c|c|}
\hline \multicolumn{2}{|c|}{ Parâmetros de Gravação } & \multirow{2}{*}{$\begin{array}{c}\text { Profundidade da oitava } \\
\text { camada } \\
\text { Profundidade da nona } \\
\text { camada }\end{array}$} & \multirow{2}{*}{$\begin{array}{l}3126 \mathrm{~m} \\
3650 \mathrm{~m}\end{array}$} \\
\hline Ângulo de Partida & $0,0594 \mathrm{rad}$ & & \\
\hline Tempo de gravação & $2,6825 \mathrm{~s}$ & $\begin{array}{c}\text { Profundidade da décima } \\
\text { camada }\end{array}$ & $3998 \mathrm{~m}$ \\
\hline $\begin{array}{c}\text { Velocidade da onda } \\
\text { na água }\end{array}$ & $1500 \mathrm{~m} / \mathrm{s}$ & $\begin{array}{c}\text { Profundidade da décima } \\
\text { primeira camada }\end{array}$ & $4440 \mathrm{~m}$ \\
\hline Densidade da água & $1000 \mathrm{~kg} / \mathrm{m}^{3}$ & $\begin{array}{l}\text { Profundidade da décima } \\
\text { segunda camada }\end{array}$ & $4886 \mathrm{~m}$ \\
\hline
\end{tabular}
. 


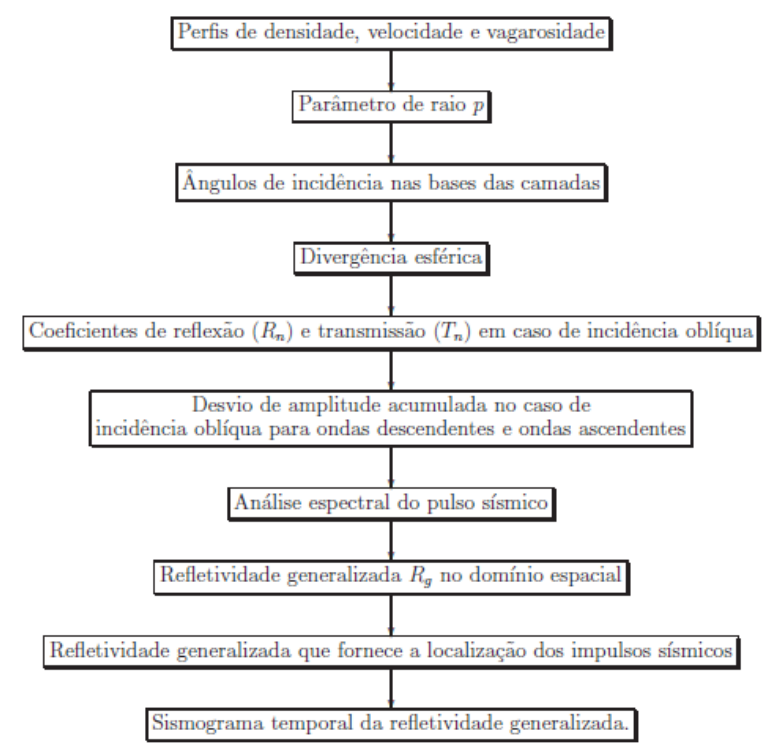

Fluxograma 1 - Modelagem sísmica para o decaimento do campo de ondas.

Os coeficientes de reflexão e transmissão no caso de incidência normal para o modelo sintético geraram o resultado mostrado na Figura 3.

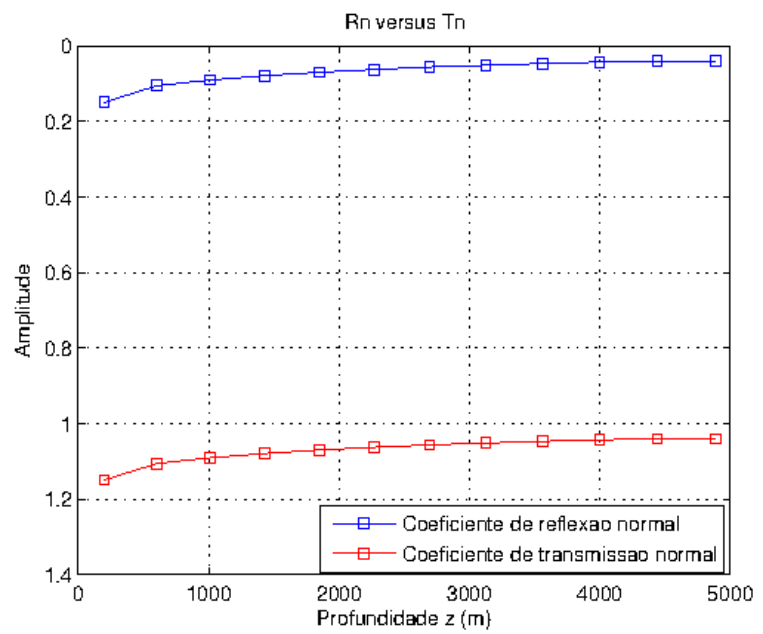

Figura 2 - Coeficientes de reflexão e transmissão normal. Na parte superior, os coeficientes de reflexão normal. Na parte inferior, os coeficientes de transmissão normal.

No caso de Incidência oblíqua, o resultado dos cálculos dos coeficientes de reflexão e transmissão está evidenciado na figura 4.

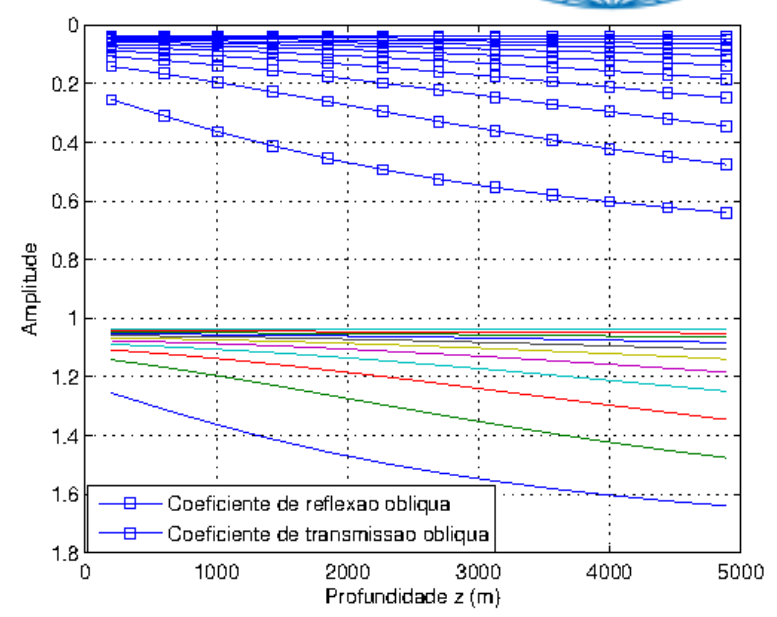

Figura 3 - Coeficientes de reflexão e transmissão oblíqua. Na parte superior, os coeficientes de reflexão oblíqua. $\mathrm{Na}$ parte inferior, os coeficientes de transmissão oblíqua.

Os desvios de amplitude acumulada no caso de incidência oblíqua para ondas descendentes e ascendentes, dados pelas Eqs. (8) e (9), geraram o resultado da Figura 4.

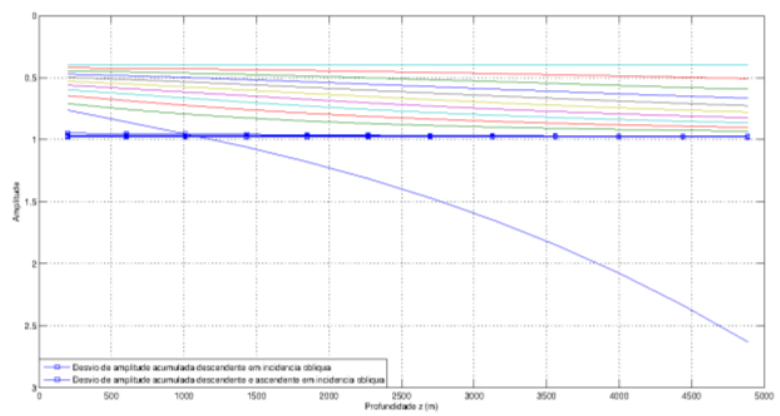

Figura 4 - Desvio de amplitude acumulada descendente e ascendente em caso de incidência oblíqua.

Para modelarmos o sismograma sintético, devemos primeiramente, construir o pulso fonte. Neste caso escolhemos o pulso Ricker, dado por

$R(t)=\left(1-2 \pi^{2} \mathrm{f}_{D O M}^{2} \mathrm{t}^{2}\right) \mathrm{e}^{-\pi^{2} \mathrm{f}_{D O M}^{2} \mathrm{t}^{2}}$

em que $\mathrm{f}_{D O M}$ é a frequência dominante do pulso, que neste caso, é de $60 \mathrm{~Hz}$, e t é o tempo de amostragem. O pulso Ricker está representado na Figura 5. 


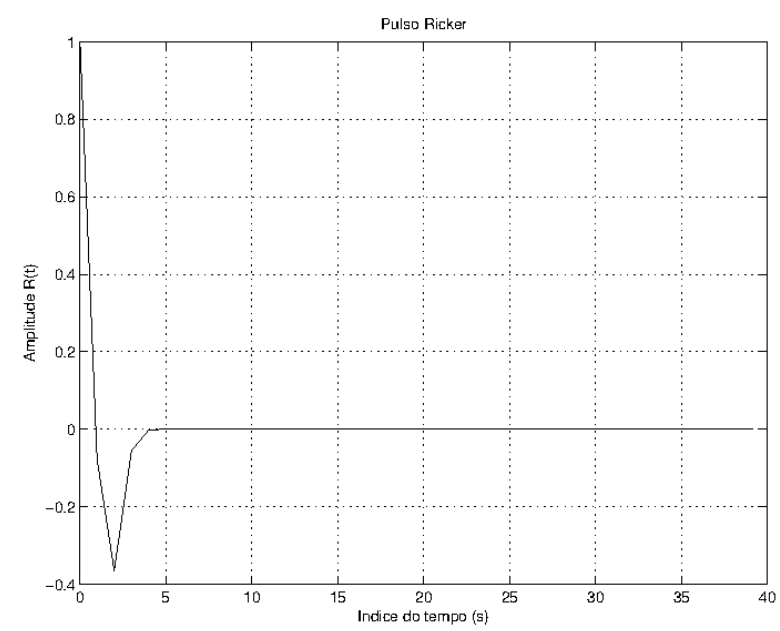

Figura 5 - Pulso Ricker, contendo 40 pontos, com incremento de tempo de $0.004 \mathrm{~s}$, usado para simular o pulso sísmico.

Para realizarmos a construção do sismograma, inicialmente calculamos a refletividade generalizada $R_{g}$ no domínio espacial para o modelo sintético em questão. Esta operação é feita segundo a equação (14).

$R_{g}=\frac{\bar{r}_{i}^{B}}{R_{0}}$

Na qual $\bar{r}_{i}^{B}$ é o desvio de amplitude acumulada no caso de incidência oblíqua para ondas descendentes e ascendentes descrito na equação (12) e $R_{0}$ corresponde ao fator de divergência esférica, descrito na equação (2).

A matriz de refletividade generalizada que fornece a localização dos impulsos temporais gera a disposição espacial dos impulsos temporais na seção sísmica. O resultado desta operação está mostrado na Figura 6 para o modelo.

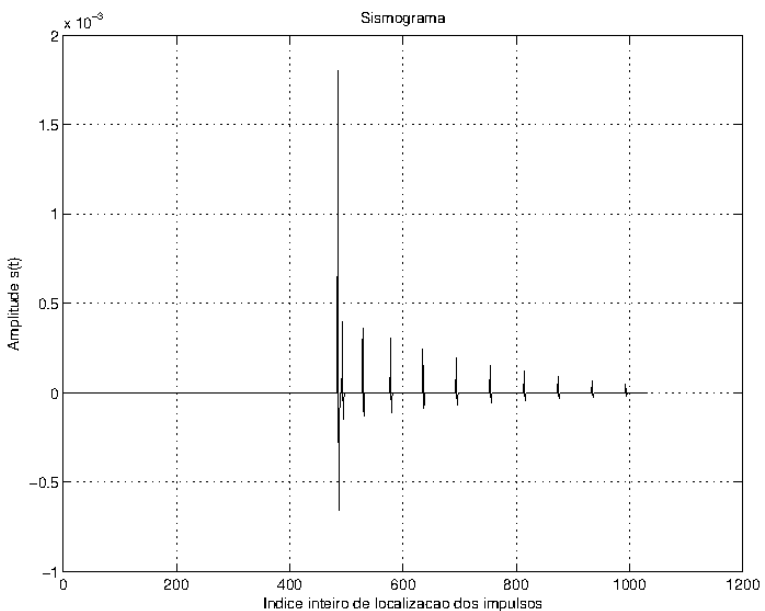

Figura 6 - Refletividade dos pulsos sísmicos com ganho no domínio temporal para o modelo.

\section{Discussão e Conclusões}

A avaliação do decaimento de amplitude de ondas sísmicas para o caso de modelo geológico de camadas plano-estratificadas mostrou-se coerente com os resultados obtidos em casos reais. Os resultados obtidos para desvios de amplitude das ondas ascendentes e descendentes em caso de incidência oblíqua foram satisfatórios, pois são da mesma ordem de amplitude dos casos obtidos em dados reais marinhos. A refletividade generalizada, gerou resultados coerentes. Os índices de localização dos impulsos, posteriormente convolvidos com o pulso Ricker, geraram finalmente o sismograma desejado, evidenciando que a onda sísmica sofre forte atenuação após ser refletida na primeira camada, sendo necessária a aplicação de funções de ganho para recuperar amplitudes muito pequenas na parte inferior dos sismogramas dos dados reais. Baseado nessas observações,portanto, pode-se afirmar que o sismograma obtido é coerente com os levantamentos reais de campo.

\section{Agradecimentos}

Os autores agradecem à Universidade Federal do Oeste do Pará - UFOPA pelo apoio logístico necessário para realização desta pesquisa.

\section{Referências}

AKI, K.; RICHARDS, P. Quantitative seismology: Theory and methods. [S.I.]: Freeman and Co., 19802002.

BARTOLO L.; DORS, C. M. W. M. C. d. S. G. D. Evaluation of the infuence of density in the acoustic waves amplitudes. Brasilian Geophysical Society, Rio de Janeiro, Brazil, v. 7, 2011.

GUTENBERG, B. The amplitudes of waves to be expected in seismic prospecting. Geophysics, v. 1, p. 252-256, June 1936.

HUBRAL, P.; KREY, T. Interval Velocities from Seismic Reflection Time Measurements. Tulsa, OK: Society of Exploration Geophysicists, 1980.

NEWMAN, P. Divergence effects in a layered earth. Geophysics., v. 38, n. 3, p. 481-488, 1973.

ROBINSON, E. A.; TREITEL, S. Geophysical signal analysis. Tulsa, OK: Society of Exploration Geophysicists, 2000. 278-279 p.

SANDMEIER, K.-J. Untersuchung der ausbreitungseigenschaften seismischer wellen in geschichteten und streuenden medien Alemanha, Instituto de Geofísica da Universidade de Karlsruhe., Tese de Doutorado, 1990. Tese (Doutorado) - Delft University of Technology, Karlsruhe, 1990.

TANER, M. T.; KOEHLER, F. Velocity spectra-digital computer derivation and applications of velocity functions. Geophysics, Tulsa, OK, v. 34, n. 6, p. 859$881,1969$. 